\section{Measuring up the planet}

\section{Barry Parsons}

Geophysical Geodesy: The Slow Deformations of the Earth. By Kurt Lambeck. Clarendon: 1988. Pp.718. Hbk £75, \$95; pbk £30.

GeOdesy, which can claim to be the oldest of the geophysical sciences, maintains a somewhat different relationship to geophysics than the other geophysical sciences. The choice of Geophysical Geodesy as a title to emphasize that this book is about the geophysical applications of geodesy points to the usually dominant role of applied aspects of the subject. In contrast the title of a book on seismology would probably only be qualified if it were about exploration or engineering seismology.

This difference can be explained in part by the long timescales involved in the development of many geodetic techniques. Consider the example of verylong-baseline interferometry (VLBI), which is the method of determining the distance between two points on the Earth's surface by using radio telescopes at the two sites to measure the small differences in arrival time of signals from distant radio sources. It has taken 20 years of painstaking development for VLBI to get to the stage where it is possible to measure directly the relative motion between the tectonic plates. Although most geophysicists appreciate the potential of many geodetic measurements, their interest is only really aroused when it becomes clear that worthwhile information about the Earth will be forthcoming.

Fortunately for those interested in the geophysical applications of geodesy, in recent years several geodetic techniques have emerged that have either already demonstrated their value in studying the Earth or seem about to do so. Satellite altimeter measurements of the shape of the ocean surface have replaced direct measurements of gravity as the primary source of information about the gravity field over the oceans. These observations provide one of the few ways of looking at the effects of mantle convection beneath the oceanic lithosphere. In the next few years the Global Positioning System will become fully operational, and this method of measuring baseline lengths, which is similar to VLBI except that satellite radio sources rather than extraterrestrial ones are used, has the potential to determine exactly how crustal deformation in the tectonically active regions of continents takes place. The nature of the core-mantle boundary is the subject of much debate, and VLBI observations of the nutations of the Earth's rotation axis allow estimates to be made of the departure of the ellipticity of the core-mantle boundary from the ellipticity expected due to the Earth's rotation alone.

Against this background, Kurt Lambeck has set himself the task of writing an account of the subject that encompasses not only the geodetic techniques but the geophysical applications as well. The breadth of material he covers is impressive, and the book will provide an excellent overview of the field for both students and research workers. Such a comprehensive treatment within a single volume has its drawbacks, however. The author has to assume that the intended audience of graduate students will already have been exposed to the basic ideas of both geodesy and geophysics, an assumption that will only be true for the more advanced

\section{Today's data}

\section{Gunnar von Heijne}

\section{Computational Molecular Biology:} Sources and Methods for Sequence Analysis. Edited by Arthur M. Lesk. Oxford University Press: 1988. Pp.254. £25, $\$ 49.95$.

FIVF years ago, in the typical molecular biology laboratory, there would be at least one computer whiz kid: a graduate student who stole time away from the bench to break into the central campus computer and entertain himself with adventure games. This student was often quickly assigned the more serious job of taking care of the sequence-analysis needs of the lab, installing the newly purchased software that no one else had found the time to get running. Thus everybody was happy: the student was promoted to the status of computer expert, and the 'real' scientists didn't have to soil their hands at the PC keyboard.

Times have changed. Even the staunchest 'wet' experimentalist has had to adapt to the fact that these days one simply cannot do molecular biology without occasional recourse to the help of a computer. This, of course, is mainly a result of the extraordinary growth of DNA sequence data over the past ten years: more than 20 million bases worth of sequence is now stored in the main data banks such as GenBank or the European Molecular Biology Laboratory's data library, and only computers can deal with such massive amounts of information.

The world of 'machine molecular biology' is not immediately accessible to the average biologist, however, and good introductory texts are badly needed. The so-called CODATA Task Group on Coordination of Protein Sequence Data student. In addition, by introducing many results without the concomitant theoretical development, one loses a sense of the physics that lies behind the equations.

The subtitle, The Slow Deformations of the Earth, reflects the author's interests and the contents of the book are clearly weighted towards solid-Earth geophysics. Physical oceanography, which will be the principal beneficiary of the two satellite altimeter missions due over the next five years, appears in a subordinate role. Yet. with the broad range of geophysical applications that are included, and its appearance just as new observations are about to flood in, this book is bound to attract a wide audience.

Barry Parsons is Reader in Geodesy in the Department of Earth Sciences, University of Oxford, Parks Road, Oxford OX1 3PR, UK

Banks has now put out a volume called Computational Molecular Biology, edited by Arthur Lesk, to meet the demand.

The book is organized around four questions - What data are available? How can one gain access to them and to the necessary programs? What calculations can be done? And how can the results of the calculations be intelligently and cautiously interpreted? The first and second questions are treated at length; the third and fourth are less fully explored. We thus learn a lot about a number of DNA- and protein-sequence data banks around the glohe, and we are introduced to the hardware and software of sequence analysis as well as to some aspects of computer networking. But we are told less about the actual methods and algorithms that allow the computer to produce the output we want.

Lesk apparently sensed this bias at an early stage, and has written a number of the less 'computerish' chapters himself: chapters for people who care more about how to find probable coding regions, or predict the structure of a protein, than about the details of how a GenBank entry is organized. Lesk has also come up with a couple of remarks that I will cherish for a long time to come. Thus, in summing up secondary structure prediction for proteins, he delivers the following: "Running a secondary structure prediction on a newly-determined sequence just because everyone else does so, is to be deplored, and the fact that the results of such predictions are generally ignored is insufficient justification for doing and publishing them". This is the sort of text that should be printed on every sequence-analysis software diskette as a 'Warning from the Surgeon General'.

Gunnar von Heijne is in the Department of Molecular Biology, Karolinska Institute Center for Biotechnology. Huddinge University Hospital, S-141 86 Huddinge, Sweden. 